\title{
Performance Analysis of Industrial Cooperative Communication System in Generalized Fading Environment
}

\author{
Ivica MARJANOVIC, Nenad MILOSEVIC, Aleksandra CVETKOVIC, Dejan MILIC, Zorica NIKOLIC
}

\begin{abstract}
This paper considers the performance analysis of M-ary phase shift keying industrial cooperative relaying system in Nakagami-m multipath fading channel with Gamma shadowing, which is known as the Generalized-K composite fading channel. Since the paper deals with the industrial environment, the communication channel is also affected by the additive Middleton's Class-A impulsive noise. The bit error rate is used as a performance measure and the closed-form average bit error rate expression was derived. The influence of different fading and noise parameters on the system performance is investigated. The obtained results may be used for the improvement of the present and future industrial wireless communication systems.
\end{abstract}

Keywords: bit error rate; Generalized-K fading; Impulsive noise; M-ary phase shift keying

\section{INTRODUCTION}

Wireless Sensor Networks (WSN's) are increasingly popular over the past two decades and today they are significantly more used for different sensing applications then wired networks $[1,2]$. A subclass of WSN's for the application in the industrial environment is known as Industrial WSN's (IWSN's). The number of devices in IWSN's increased more than five times during the past five years [3] and is expected to increase even more in the future. With the development of Internet of Things (IoT), WSNs became even more important and more interesting for the research. IoT represents a network of devices with smart capabilities that are connected via a communication channel [4]. Main elements of IoT networks are sensors and therefore WSNs are and will be very important for these networks [5]. There is an estimate of 50 billion active IoT devices by 2020 [6], and most of them will have actuators and/or sensors, which also confirms the importance of future WSN networks. At the same time, the Industrial Internet or Industry 4.0 [7] is in rapid development but it is still a relatively immature concept. Industry 4.0 will initiate growth and improvement of industrial infrastructure with the introduction of novel principles that will be based on the information and communication technology.

Since devices within WNS operate with limited energy, it is of highest importance to implement communication schemes with low energy consumption. The energy needed for the communication may be reduced by shortening the distance between two devices. This goal may be achieved with the introduction of the cooperative communications, where the third WSN node serves as a relay for the first two nodes [8-10]. Besides, the relayed transmission may also improve the coverage of the network. Depending on the strategy of the relaying node, there are two different relaying categories: amplify-andforward (AF), when the relaying node just amplifies and forwards the received signal, and decode-and-forward (DF) when the relay fully decodes the received messages, encodes them again and also forwards towards the next relay or destination [11-13]. A lot of research has been done regarding the analysis of relaying systems with AF and DF schemes, for different communication channels [14-19]. Besides the small-scale multipath fading, the IWSNs communication channels are prone to the slow variation of the mean signal level due to the shadowing from obstacles. The effects of the large-scale shadowing in WSN's are analyzed in a number of papers [20-23].

Besides fading, IWSN channels are characterized by the impulsive noise, also. Namely, there are a lot of sources of the impulsive noise in the industrial environment, such as electro motors, electromechanical switches and other industrial devices $[24,25]$. However, the impulsive noise is often neglected in the literature and the noise is treated as AWGN. The importance of treating industrial noise in a proper way is shown in [26], where the impulsive noise is modelled as Middleton's Class-A (MCA) model, which is in great accordance with practical measurements in the industrial environments [27-29].

Having in mind all of the above, a DF relaying scheme for the IWSNs employing MPSK (M-ary Phase Shift Keying) modulation is analyzed in this paper. Paper [26] analyzes relaying in IWSN's Nakagami-m fading channel and impulsive noise, but it does not take into account largescale shadowing, which is an important effect that should not be neglected. Therefore, this paper considers Nakagami-m fading channel with log-normal shadowing, in the presence of the impulsive noise.

The rest of the paper is organized as follows. Section 2 presents the model of the system. The performance analysis is given in Section 3, the numerical results in Section 4, and the conclusions in Section 5.

\section{SYSTEM MODEL}

We consider a relaying system, shown in Fig. 1, with $L+1$ nodes: the source node, $L-1$ relays and the destination node. The node $\mathrm{R}_{l}, l=1, \ldots, L$, transmits signal $s_{l}$ (with energy $E_{b}$ ) with the power $P_{l}$. The received signal at the node $\mathrm{R}_{l+1}$ is equal to

$y_{l+1}=\sqrt{P_{l}} h_{l} s_{l}+z_{R, l}, l=1, \ldots, L$

where $h_{l}$ is the fading envelope between nodes $\mathrm{R}_{l}$ and $\mathrm{R}_{l+1}$, and $z_{R, l}$ is the additive noise at node $\mathrm{R}_{l+1}$. The transmitted signal uses MPSK modulation with $M$ modulation levels.

The received signal-to-noise ratio (SNR) at the node $\mathrm{R}_{l+1}$ is defined as 
$\gamma_{l}=P_{l}\left|h_{l}\right|^{2} E_{b} / N_{0}$

where $N_{0}$ is the average power of the additive noise, defined later in (7)

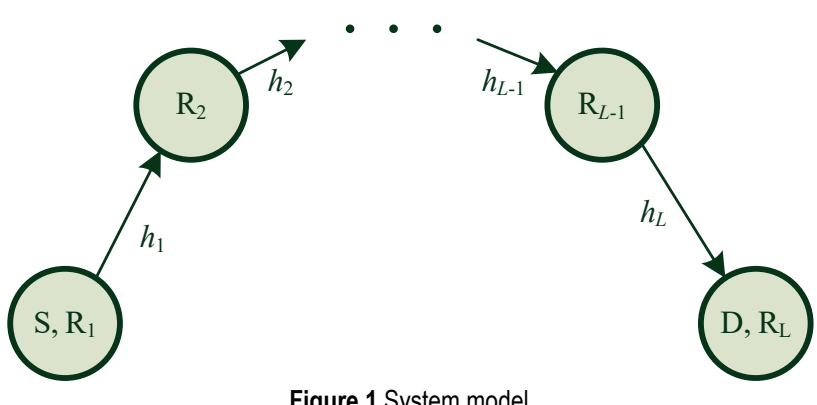

Figure 1 System model

At each of the relaying nodes, the signal is decoded, encoded again and forwarded towards the next relay or destination.

We consider the propagation channel with the effects of shadowing and multipath fading. This results in the composite fading channel model. In wireless communications, Nakagami-m multipath fading is usually used, while the log-normal distribution is used for the description of the shadowing effect. But, for the sake of more convenient mathematical analysis, Gamma distribution is often used as a model for the shadowing, which leads to Generalized- $K$ fading model [30]. Hence, the probability density function (PDF) of the instantaneous SNR at $l$-th link is given by [31]

$$
\begin{aligned}
& p_{\gamma_{l}}(\gamma)=\frac{2}{\Gamma\left(m_{m, l}\right) \Gamma\left(m_{s, l}\right)}\left(\frac{m_{m, l} m_{s, l}}{\bar{\gamma}_{l}}\right)^{\frac{m_{m, l}+m_{s, l}}{2}} . \\
& \cdot \gamma \frac{m_{m, l}+m_{s, l}}{2}-1 K_{m_{s, l}-m_{m, l}}\left(2 \sqrt{\frac{m_{m, l} m_{s, l}}{\bar{\gamma}_{l}}} \gamma\right), l=1, \ldots, L
\end{aligned}
$$

where $\Gamma(\cdot)$ denotes Gamma function [31, Eq. (8.31)], $K_{\beta}(\cdot)$ is the $\beta$-th order modified Bessel function of the second kind [32, Eq. (8.432.3)], while the multipath fading and shadowing parameters are $m_{m, l}$ and $m_{s, l}$, respectively.

The additive noise from (1) is modelled at each link as MCA identical and independent random variable. The PDF of $z_{R, l}$ is given by the weighted sum of normal distributions [33]:

$p_{z_{R, l}}(z)=\sum_{n=0}^{\infty} \frac{\alpha_{n}}{\sqrt{2 \pi} \sigma_{n}} \exp \left(-\frac{z^{2}}{2 \sigma_{n}^{2}}\right)$

where parameter $\alpha_{n}$ is equal to

$$
\alpha_{n}=\frac{e^{-A} A^{n}}{n !}
$$

The variance $\sigma_{n}^{2}$ is defined as:

$$
\sigma_{n}^{2}=N_{0} \frac{(n+A \cdot \rho)}{A \cdot(1+\rho)}
$$

with $\rho=\sigma_{g}^{2} / \sigma_{i}^{2}$, where $\sigma_{g}^{2}$ and $\sigma_{i}^{2}$ are the variances of the Gaussian and impulsive noise, respectiveliy. It was shown in [26] that the average power of the noise is:

$$
N_{0}=\sigma_{g}^{2}+\sigma_{i}^{2}
$$

Parameter $A$ defines the impulsiveness of the noise: the lower the value the more impulsive the noise is. In case of $A \rightarrow \infty$, MCA impulsive noise does not have the impulsive component and becomes pure white Gaussian noise.

\section{PERFORMANCE ANALYSIS}

The bite error rate of the $l$ th link, conditioned on SNR $\gamma$, may be expressed as [34]:

$P_{l}(e \mid \gamma)=\sum_{n=0}^{\infty} \frac{2 \alpha_{n}}{\varsigma_{M}} \sum_{i=1}^{\max \left(\frac{M}{4}, 1\right)} Q\left(\sqrt{2 \log _{2} M \cdot \frac{A \gamma(1+\rho)}{(n+A \rho)}} \eta_{i}\right)$

The average BER is equal to

$P_{l}=\int_{0}^{\infty} P_{l}(e \mid \gamma) p_{\gamma l}(\gamma) d \gamma$

After substituting (3) in (8), we get

$$
\begin{aligned}
& P_{l}=\frac{2}{\Gamma\left(m_{m, l}\right) \Gamma\left(m_{s, l}\right)}\left(\frac{m_{m, l} m_{s, l}}{\bar{\gamma}_{l}}\right)^{\frac{m_{m, l}+m_{s, l}}{2}} . \\
& \cdot \sum_{n=0}^{\infty} \sum_{i=1}^{\max \left(\frac{M}{4}, 1\right)} \alpha_{n} \frac{2}{\varsigma_{M}} \times \int_{0}^{\infty} \gamma \frac{m_{m, l}+m_{s, l}}{2}-1 . \\
& \cdot Q\left(\sqrt{2 \log _{2} M \cdot \frac{A \gamma(1+\rho)}{(n+A \rho)}} \eta_{i}\right) . \\
& \cdot K_{m_{s, l}-m_{m, l}}\left(2 \sqrt{\frac{m_{m, l} m_{s, l}}{\bar{\gamma}_{l}} \gamma}\right) d \gamma
\end{aligned}
$$

Having in mind that $Q(\sqrt{x})=\frac{1}{2} \operatorname{erfc}(\sqrt{x / 2})$ and using [35, Eq. (03.04.26.0009.01)]:

$$
K_{v}(\sqrt{z})=\frac{1}{2} G_{0,2}^{2,0}\left(\frac{z}{4} \mid \frac{v}{2},-\frac{v}{2}\right)
$$

and [36, Eq. (06.27.26.0006.01)]:

$\operatorname{erfc}(\sqrt{z})=\frac{1}{\sqrt{\pi}} G_{1,2}^{2,0}\left(\begin{array}{c}1 \\ z \\ 0, \frac{1}{2}\end{array}\right)$

we get 


$$
\begin{aligned}
& P_{l}=\frac{1}{\sqrt{\pi} \Gamma\left(m_{m, l}\right) \Gamma\left(m_{s, l}\right) \varsigma_{M}}\left(\frac{m_{m, l} m_{s, l}}{\bar{\gamma}_{l}}\right)^{\frac{m_{m, l}+m_{s, l}}{2}} . \\
& \cdot \sum_{n=0}^{\infty} \sum_{i=1}^{\max \left(\frac{M}{4}, 1\right)} \alpha_{n} \text {. } \\
& \cdot \int_{0}^{\infty} \gamma^{\frac{m_{m, l}+m_{s, l}}{2}-1} G_{1,2}^{2,0}\left(\log _{2} M \cdot \frac{A(1+\rho) \eta_{i}^{2}}{(n+A \rho)} \gamma \mid \begin{array}{c}
1 \\
0, \frac{1}{2}
\end{array}\right) . \\
& \cdot G_{0,2}^{2,0}\left(\frac{m_{m, l} m_{s, l}}{\bar{\gamma}_{l}} \gamma \mid \frac{m_{s, l}-m_{m, l}}{2}, \frac{m_{m, l}-m_{s, l}}{2}\right) d \gamma
\end{aligned}
$$

where $G_{p, q}^{m, n}(\cdot)$ denotes Meijer's G-function [32, Eq. (9.301)].

After using the identity [37, Eq. (07.34.21.0011.01)] we get the final closed-form expression for the average BER of the $l$-th link:

$$
\begin{aligned}
& P_{l}=\frac{1}{\sqrt{\pi} \Gamma\left(m_{m, l}\right) \Gamma\left(m_{s, l}\right) \varsigma_{M}} \sum_{n=0}^{\infty} \sum_{i=1}^{\max \left(\frac{M}{4}, 1\right)} \alpha_{n} . \\
& \cdot G_{2,3}^{2,2}\left(\frac{m_{m, l} m_{s, l}(n+A \rho)}{\bar{\gamma}_{l} \log _{2} M \cdot A(1+\rho) \eta_{i}^{2}} \mid \begin{array}{c}
1, \frac{1}{2} \\
m_{s, l}, m_{m, l}, 0
\end{array}\right)
\end{aligned}
$$

In order to calculate the average BER for the entire system, from the source to the destination, without the loss of generality and for the sake of clarity, we will assume that all links are equal:

$$
\begin{aligned}
& m_{m, 1}=m_{m, 2}=\cdots=m_{m, L}=m_{m} \\
& m_{s, 1}=m_{s, 2}=\cdots=m_{s, L}=m_{s} \\
& \bar{\gamma}_{1}=\bar{\gamma}_{2}=\cdots=\bar{\gamma}_{L}
\end{aligned}
$$

Now, the average BER may be expressed as:

$$
P_{t o t}=\frac{1}{2}\left[1-(1-2 P)^{L}\right]
$$

where $P_{1}=P_{2}=\ldots=P_{L}=\mathrm{P}$.

\section{NUMERICAL RESULTS}

In this section we present some numerical results that indicate the influence of the fading and noise parameters on the bit error probability. The derived theoretical expressions are confirmed by the Monte-Carlo simulations.

Fig. 2 represents the bit error probability as a function of the average SNR for different MCA impulsive index $A$ and BPSK modulation. Noise parameter $\rho$ and fading parameters $m_{m}$ and $m_{s}$ are fixed. The noise parameter $A$ is chosen to model different noise conditions, from very impulsive $\left(A=10^{-5}\right)$ to almost non-impulsive $(A=10)$. It can be seen that the theoretical results are in great accordance with the simulations. As shown in [26] and explained in [38], for low values of $A$, BER curve has three areas: for low and high SNR values, BER changes more rapidly with SNR, than in the case of moderate SNR values.

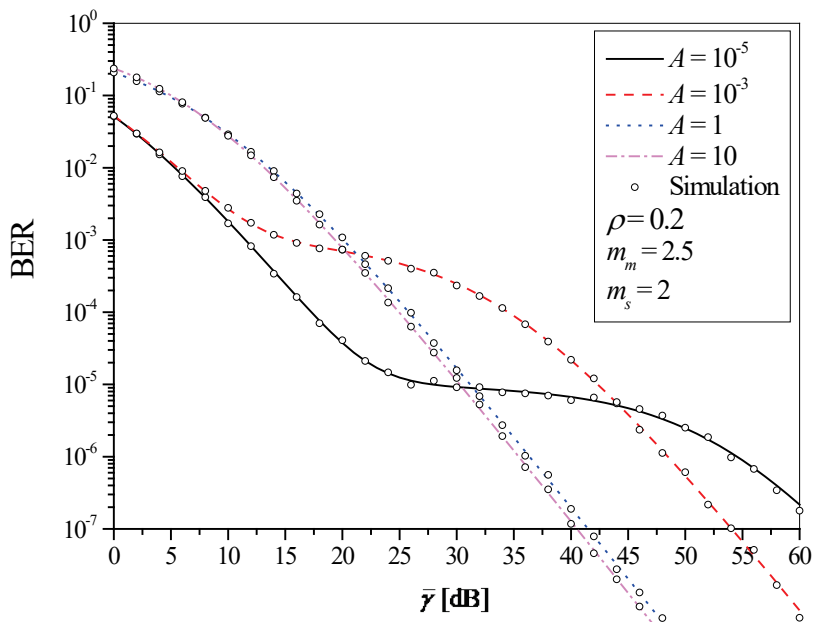

Figure 2 The average BER as a function of average SNR for several noise parameters $A$ and BPSK

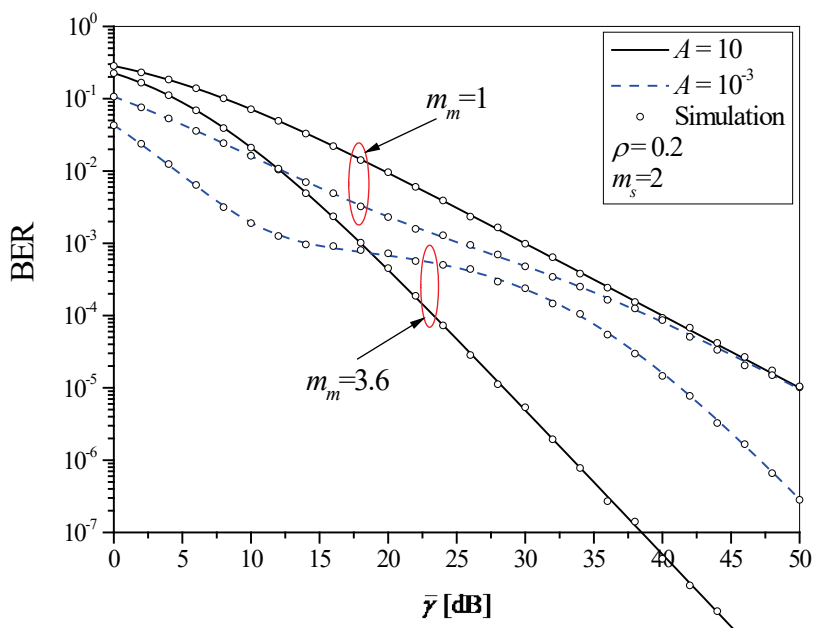

Figure 3 The average BER as a function of average SNR for different fading parameters and BPSK

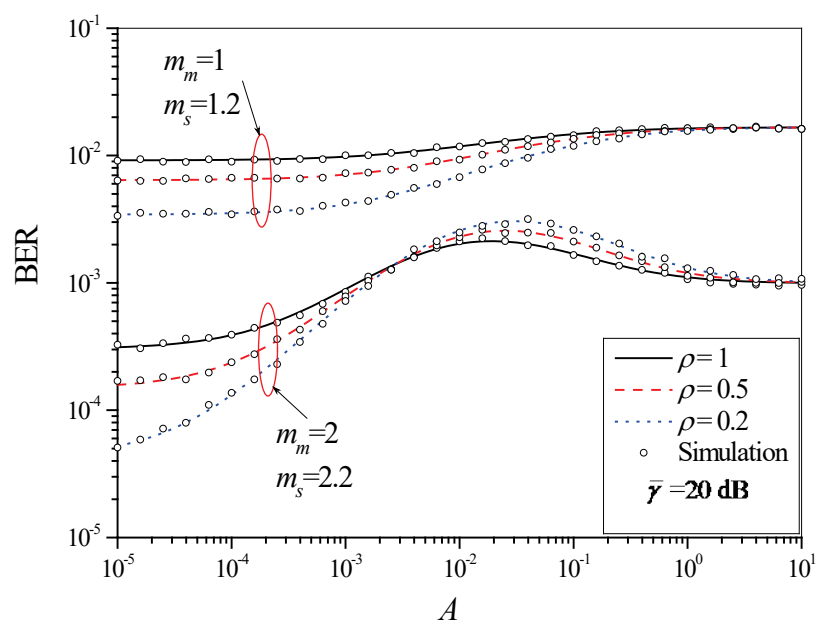

Figure 4 The average BER as a function of noise parameter $A$ for BPSK

By proper choice of the proposed fading channel parameters, it is possible to demonstrate different wellknown channel conditions: for high values of the shadowing parameter $m_{s}$, the channel becomes Nakagami-m. Also, for $m_{m}=1$, Nakagami-m channel simplifies to Rayleigh fading channel. On the other hand, high $m_{s}$ and high $m_{m}$ produce no 
fading conditions. Therefore, Fig. 3 shows BER performance for different channel conditions: from the Generalized-K channel with MCA impulsive noise, to the pure AWGN channel without fading.

The influence of impulsive index $(A)$ and the ratio of the Gaussian noise power and the impulsive noise power $(\rho)$ on the bit error rate is shown in Fig. 4 for $\mathrm{SNR}=20 \mathrm{~dB}$. The results show that in case of more severe fading conditions $\left(m_{m}=1\right.$ and $\left.m_{s}=1.2\right)$ BER increases with the increase of $A$ and with the increase of $\rho$. In contrast, for less severe fading conditions $\left(m_{m}=2\right.$ and $\left.m_{s}=2.2\right)$, BER reaches its maximum for a moderate value of $A$ around 0.03 . Furthermore, for low $A$, BER is higher for higher $\rho$, and for moderate $A$, BER is lower for higher $\rho$.

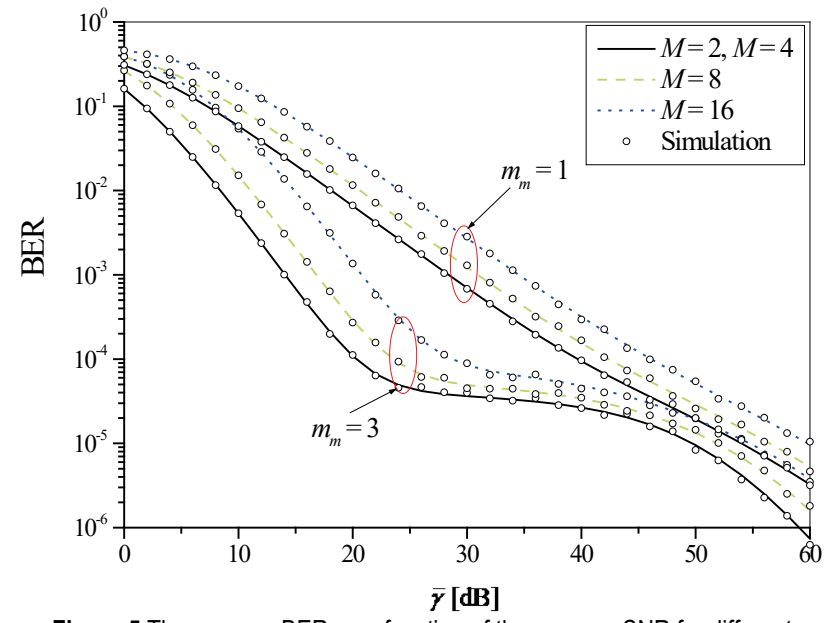

Figure 5 The average BER as a function of the average SNR for different numbers of modulation levels

Fig. 5 shows the influence of the number of MPSK modulation levels $(M)$ on the average BER for $L=8$ hop network. The results confirm a known theoretical property that the BER is the same for BPSK and 4PSK. Besides, SNR and the fading parameter mm influence the error probability in the same way, regardless of the number of modulation levels.

\section{CONCLUSION}

Industrial WSNs are gaining in importance on one hand, and the cooperative communications are important for wireless sensors networks due to lower energy usage. Therefore, this paper investigates bit error rate of a cooperative industrial WSN with impulsive noise in communication channel with the effects of both multipath fading and shadowing. A closed-form expression for the bit error rate is derived, and it was used to examine the impact of fading parameters (severity of multipath fading $m_{m}$ and shadowing $m_{s}$ ), the Middleton's Class-A impulsive noise parameters (impulsive index $A$ and the ratio of the noise Gaussian component power to the impulsive component power $\rho$ ) and the number of MPSK modulation levels $M$ on the bit error rate of the system. The theoretical results are shown to be in great accordance with the Monte-Carlo simulation. The obtained results show the most important parameters that need to be taken care of during the design of the industrial wireless sensor networks.

\section{Acknowledgements}

The research leading to these results has received funding from the Ministry of Education, Science and Technological Development of Serbia within the projects TR-32051 and TR-32037.

\section{REFERENCES}

[1] Pottie, G. J. (2016). Wireless Sensor Networks Concepts, Applications, Experimentation and Analysis. 1998 Information Theory Workshop (Cat. No.98EX131). https://doi.org/10.1109/INFOCOM.2014.6848209

[2] Swami, A., Zhao, Q., Hong, Y.-W., \& Tong, L. (2007). Wireless sensor networks: signal processing and communications perspectives. John Wiley \& Sons.

[3] Hatler, M. (2012). Industrial Wireless Sensor Networks: Trends and developments. InTech Magazine. Retrieved from https://www.isa.org/standards-publications/isapublications/intech-magazine/2012/october/web-exclusiveindustrial-wireless-sensor-networks

[4] Gubbi, J., Buyya, R., Marusic, S., \& Palaniswami, M. (2013). Internet of Things (IoT): A vision, architectural elements, and future directions. Future Generation Computer Systems, 29(7), 1645-1660. https://doi.org/10.1016/j.future.2013.01.010

[5] Jacobsson, M. \& Orfanidis, C. (2015). Using softwaredefined networking principles for wireless sensor networks. In 11th Swedish National Computer Networking Workshop (SNCNW 2015) Karlstad, May 28-29, 2015.

[6] Cisco. (2016). Fog Computing and the Internet of Things: Extend the Cloud to Where the Things are. Cisco White Paper. San Jose, CA, USA.

[7] Platform Industrie 4.0. (n.d.). Retrieved November 1, 2018, from https://www.plattform-i40.de/I40/Navigation/EN/ Home/home.html

[8] Sendonaris, A., Erkip, E., \& Aazhang, B. (1998). Increasing uplink capacity via user cooperation diversity. In Proceedings. 1998 IEEE International Symposium on Information Theory (Cat. No.98CH36252), 156. https://doi.org/10.1109/ISIT.1998.708750

[9] Hasna, M. O. \& Alouini, M. (2002). Performance analysis of two-hop relayed transmissions over Rayleigh fading channels. In Proceedings IEEE $56^{\text {th }}$ Vehicular Technology Conference, 4, 1992-1996. https://doi.org/10.1109/VETECF.2002.1040567

[10] Hasna, M. O. \& Alouini, M. (2004). Optimal power allocation for relayed transmissions over Rayleigh-fading channels. IEEE Transactions on Wireless Communications, 3(6), 1999-2004. https://doi.org/10.1109/TWC.2004.833447

[11] Nosratinia, A., Hunter, T. E., \& Hedayat, A. (2004). Cooperative communication in wireless networks. IEEE Communications Magazine, 42(10), 74-80. https://doi.org/10.1109/MCOM.2004.1341264

[12] Kramer, G., Gastpar, M., \& Gupta, P. (2005). Cooperative strategies and capacity theorems for relay networks. IEEE Transactions on Information Theory, 51(9), 3037-3063. https://doi.org/10.1109/TIT.2005.853304

[13] Islam, S. N., Sadeghi, P., \& Durrani, S. (2013). Error performance analysis of decode-and-forward and amplifyand-forward multi-way relay networks with binary phase shift keying modulation. IET Communications, 7(15), 16051616. https://doi.org/10.1049/iet-com.2013.0284

[14] Dhaka, K., Mallik, R. K., \& Schober, R. (2012). Performance Analysis of Decode-and-Forward Multi-Hop Communication: A Difference Equation Approach. IEEE Transactions on Communications, 60(2), 339-345. https://doi.org/10.1109/TCOMM.2012.12.100705

[15] Morgado, E., Mora-Jimenez, I., Vinagre, J. J., Ramos, J., \& 
Caamano, A. J. (2010). End-to-End Average BER in Multihop Wireless Networks over Fading Channels. IEEE Transactions on Wireless Communications, 9(8), 24782487. https://doi.org/10.1109/TWC.2010.070710.090240

[16] Dixit, D. \& Sahu, P. R. (2018). Error rate and outage of dualhop DF relay system with selection combining over Rice fading. International Journal of Communication Systems, 31(13), e3719. https://doi.org/10.1002/dac.3719

[17] Dixit, D. \& Sahu, P. R. (2017). Performance of dual-hop DF relaying systems with QAM schemes over mixed $\eta-\mu$ and $\kappa$ $\mu$ fading channels. Transactions on Emerging Telecommunications Technologies, 28(11), e3179. https://doi.org/10.1002/ett.3179

[18] Althunibat, S. \& Mesleh, R. (2017). Cooperative decodeand-forward quadrature spatial modulation over correlated and imperfect $\eta-\mu$ fading channels. Wireless Networks. https://doi.org/10.1007/s11276-017-1585-z

[19] Prince, A., Abdalla, A. E., Dahshan, H., \& Rohiem, A. E. (2017). Performance evaluation of multihop decode and forward cooperative relaying. In 2017 Intl Conf on Advanced Control Circuits Systems (ACCS) Systems 2017 Intl Conf on New Paradigms in Electronics Information Technology (PEIT), 321-325. https://doi.org/10.1109/ACCS-PEIT.2017.8303059

[20] Bahl, N., Sharma, A. K., \& Verma, H. K. (2014). On the energy utilization for WSN based on BPSK over the Generalized-K shadowed fading channel. Wireless Networks, 20(8), 2385-2393. https://doi.org/10.1007/s11276-014-0743-9

[21] Dziri, A., Terre, M., \& Nasser, N. (2016). Performance analysis of decode and forward relaying over Generalized-K channels at arbitrary SNR for wireless sensor networks. In 2016 International Wireless Communications and Mobile Computing Conference (IWCMC), 1100-1104. https://doi.org/10.1109//WCMC.2016.7577212

[22] Liu, B. H., Otis, B. P., Challa, S., Axon, P., Chou, C., \& Jha, S. (2008). The impact of fading and shadowing on the network performance of wireless sensor networks. International Journal of Sensor Networks, 3(4), 211-223. https://doi.org/10.1504//JSNet.2008.019006

[23] Liu, B. H., Otis, B., Challa, S., Axon, P., Chou, C. T., \& Jha, S. (2006). On the Fading and Shadowing Effects for Wireless Sensor Networks. In 2006 IEEE International Conference on Mobile Ad Hoc and Sensor Systems, 51-60. https://doi.org/10.1109/MOBHOC.2006.278597

[24] Gungor, V. C. \& Hancke, G. P. (2009). Industrial Wireless Sensor Networks: Challenges, Design Principles, and Technical Approaches. IEEE Transactions on Industrial Electronics, 56(10), 4258-4265. https://doi.org/10.1109/TIE.2009.2015754

[25] Soon, L. K., Nu, W. W. N., \& Joo, E. M. (2005). Wireless Sensor Networks for Industrial Environments. In International Conference on Computational Intelligence for Modelling, Control and Automation and International Conference on Intelligent Agents, Web Technologies and Internet Commerce (CIMCA-IAWTIC'06), 2, 271-276. https://doi.org/10.1109/CIMCA.2005.1631480

[26] Ai, Y. \& Cheffena, M. (2017). On Multi-Hop Decode-andForward Cooperative Relaying for Industrial Wireless Sensor Networks. Sensors, 17(4-695), 1-21. https://doi.org/10.3390/s17040695

[27] Middleton, D. (1983). Canonical and Quasi-Canonical Probability Models of Class a Interference. IEEE Transactions on Electromagnetic Compatibility, EMC25(2), 76-106. https://doi.org/10.1109/TEMC.1983.304151

[28] Matsumoto, Y., Wu, I., Gotoh, K., \& Ishigami, S. (2013). Measurement and modeling of electromagnetic noise from LED light bulbs. IEEE Electromagnetic Compatibility Magazine, 2(4), 58-66.

https://doi.org/10.1109/MEMC.2013.6714699
[29] Sarr, N. B., Boeglen, H., Agba, B. L., Gagnon, F., \& Vauzelle, R. (2016). Partial discharge impulsive noise in 735 $\mathrm{kV}$ electricity substations and its impacts on $2.4 \mathrm{GHz} \mathrm{ZigBee}$ communications. In 2016 International Conference on Selected Topics in Mobile Wireless Networking (MoWNeT), 1-7). https://doi.org/10.1109/MoWNet.2016.7496610

[30] Abdi, A. \& Kaveh, M. (1999). On the utility of gamma PDF in modeling shadow fading (slow fading). In 1999 IEEE $49^{\text {th }}$ Vehicular Technology Conference (Cat. No.99CH36363), 3, 2308-2312). https://doi.org/10.1109/VETEC.1999.778479

[31] Bithas, P. S., Sagias, N. C., Mathiopoulos, P. T., Karagiannidis, G. K., \& Rontogiannis, A. A. (2006). On the performance analysis of digital communications over generalized-K fading channels. IEEE Communications Letters, 10(5), 353-355. https://doi.org/10.1109/LCOMM.2006.1633320

[32] Gradstein, E. S. \& Ryzhik, I. M. (2007). Table of integrals, sums, series, and products. (A. Jeffrey \& D. Zwillinger, Eds.) $\left(7^{\text {th }}\right.$ ed.). San Diego, CA, USA: Academic Press. Retrieved from http://www.sciencedirect.com/science/ book/9780123736376

[33] Spaulding, A. \& Middleton, D. (1977). Optimum Reception in an Impulsive Interference Environment - Part I: Coherent Detection. IEEE Transactions on Communications, 25(9), 910-923. https://doi.org/10.1109/TCOM.1977.1093943

[34] Lu, J., Letaief, K. B., Chuang, J. C.-., \& Liou, M. L. (1999). M-PSK and M-QAM BER computation using signal-space concepts. IEEE Transactions on Communications, 47(2), 181-184. https://doi.org/10.1109/26.752121

[35] Wolfram Research. (2001). BesselK. Retrieved from http://functions.wolfram.com/03.04.26.0009.01

[36] Wolfram Research. (2001). Erfc. Retrieved from $\mathrm{http} / / /$ functions.wolfram.com/06.27.26.0006.01

[37] Wolfram Research. (2001). MeijerG. Retrieved from $\mathrm{http} / / /$ functions.wolfram.com/07.34.21.0011.01

[38] Zabin, S. M. \& Poor, H. V. (1989). Parameter estimation for Middleton Class A interference processes. IEEE Transactions on Communications, 37(10), 1042-1051. https://doi.org/10.1109/26.41159

\section{Contact information}

\section{Ivica MARJANOVIC}

(Corresponding author)

Military Quality Control, Ministry of Defence, Republic of Serbia

Ratka Resanovica 1, 11147 Belgrade, Serbia

ivica.marjanovic@mod.gov.rs

\section{Nenad MILOSEVIC}

University of Nis, Faculty of Electronic Engineering

Aleksandra Medvedeva 14, 18000 Nis, Serbia

nenad.milosevic@elfak.ni.ac.rs

\section{Aleksandra CVETKOVIC}

University of Nis, Faculty of Electronic Engineering

Aleksandra Medvedeva 14, 18000 Nis, Serbia

aleksandra.cvetkovic@elfak.ni.ac.rs

\section{Dejan MILIC}

University of Nis, Faculty of Electronic Engineering Aleksandra Medvedeva 14, 18000 Nis, Serbia dejan.milic@elfak.ni.ac.rs

\section{Zorica NIKOLIC}

University of Nis, Faculty of Electronic Engineering

Aleksandra Medvedeva 14, 18000 Nis, Serbia

zorica.nikolic@elfak.ni.ac.rs 